\section{Confusion about Chinese names}

SIR - Richard J. Wang's note "Xiaoping first?" $(308,768 ; 1984)$ only scratched the surface of the issue of Chinese family names in romanization. In the five articles detailing results of the French-Chinese study of the North Himalaya-Tibet area (308, 17-36; 1984), only the second has authors' names correctly abbreviated in the table of contents. Admittedly, it is difficult for the uninitiated to know (or even make an educated guess) at which is the family name.

Confusion begins because the Chinese custom in Chinese is to put family name first, the opposite of the European custom. Added confusion comes because in romanization some Chinese westernize name order (family name last) while others do not. Further, some Chinese hyphenate the given name because it is two-character (confusing at first because Europeans sometimes take a hyphenated family name), while others (particularly in mainland China) fuse the romanization into one "word". Still others make three separate words for their name, so that the given name would be two words, always to be used together. This last practice is the most confusing since there is no cue to guess the family name; in the other methods, once realized, the hyphen, or the length of the word, allows the given, and thus the family, name to be identified. Even if one is familiar with the common Chinese family names, there are several distinct, widelyused, romanization systems (so $\mathrm{Xu}=\mathrm{Hsu}=\mathrm{Shyu}$ ). Further, while pinyin is the sole romanization taught in mainland China, many Chinese elsewhere are not taught any particular system at all, so they sometimes show noticeable creativity. Knowing common last names in one system certainly helps, but not all the time.

The issue raised by all this confusion, particularly in the professional literature, is much more than that of slighting persons or cultures. It can and has already created a mess in indices. For example, to look up articles by $L i$ Tindong in an index or abstracting service, does one look up $L$. Tindong, as given in that Nature table of contents and index. Or is it T. Li? I do not think it would happen, but if an editor insisted on consistency, it could also become T.D. Li, or T.-d. Li, or T.-D. Li. What is one to do? Even the thought of tediously searching each alternative is tiring, not to mention expensive if done through computer hook-up.

As an international journal, Nature should not be making these mistakes. Even after Mr Wang's letter I found an article by Zhang Yun $(309,547 ; 1984)$ listed in the table of contents and the mid-year index (310, 26 July 84) as Yun, Z. Poor Professor Zhang! Filed under first name. Ignorance could be claimed legitimately by my nextdoor neighbour, but by Nature? Clear, fair instructions to the authors, and clear, fair editorial rules need to be established to handle these problems. Tell authors to circle family names, or, to indicate that last name, with or without hyphen, will be assumed familial unless otherwise indicated. Then give an example. Errors could be picked up, such as where R. Xu (in the first of the five articles mentioned) inverted his name apparently only because it is last in the list, even though it appears Nature's policy not to invert name order because of placement in a list of authorship. Consultation with librarians at an institute for Asian studies might help. After all, Nature does not abbreviate L.M. Van Valen as L.M.V. Valen (table of contents 5 January 1984).

I also suggest that indices should include the full romanized Chinese name. There are strong if not compelling reasons to support it. First is the lack of diversity in Chinese family names. Anyone familiar with Chinese names knows how widespread are a small number of family names such as Wang, Chen, Li, Zhou and so on. Indeed, in romanization the situation is worse than in Chinese because homophones (same sound and tone, different character) are so common. In addition, widely-used romanizations do not distinguish total differences, causing further redundancy. The only mitigating factor is

\section{Student fees in Britain}

SIR - When a nation is under financial strain, economies are necessary even in an important sector such as scientific research, which in the long run is one of the best investments. One could go further, admitting that financial restrictions, to some extent, may even be beneficial to research, in that they stimulate competition, force a better selection of people and projects and favour a more rational utilization of equipment and resources.

There is one measure, however, to which I would like to call your readers' attention, namely, the higher fees imposed on university students who are not resident in the United Kingdom. Apart from any question of reciprocity (I do not think that British students are requested to pay higher fees in any foreign university), I think that such a rule will ultimately damage British science. I know a case, by no means unique, of a British subject, not resident in the United Kingdom, who, having graduated cum laude abroad, wanted to study for a $\mathrm{PhD}$ in Britain. She would have been accepted by two leading university departments but at a cost of several thousand pounds. As a result, she accepted a research assistantship leading to a $\mathrm{PhD}$, with an adequate salary, in a department of excellent reputation in another country in the European Community.

This is only one example of how foreign that use of different romanization schemes adds some variability. Chinese given names are quite varied, but all the points on romanization apply here as well. The habit of complete fusing of the given name further reduces variability when the given name is initialized. So the combination of initialling and the commonness of most family names must lead to immense redundancy.

Finally, Chinese names are short. Most are three characters - one familial, two for the given name $-\mathbf{a}$ few such as Zhang Yun are two, though a few others are four - for each name. Thus, one would be hard-pressed to find a romanized Chinese name over 15 letters. This is not true for names in many other languages, for example N.C. Vamvakopoulous (table of contents, 5 January 1984). Nature has recognized this since Chinese names are given in full at the top of all articles. The policy needs to be extended, perhaps not to the table of contents (so people know the family name!), but definitely to indexes.

With the increasingly large numbers of Chinese names coming into the western scientific literature, there are wide ramifications for information retrieval, not to mention human dignity.

Boston University,

JAMIE HOOK

Biological Science Center,

2 Cummington Street,

Boston, Massachusetts 02215, USA

students and graduates are actually discouraged from coming to Britain. Gifted students at least, especially at postgraduate level, should be attracted, even at the cost of some money. First of all, graduate students do productive work, which would be very cheap indeed if the nation has not borne the cost of their previous support and education. Secondly, when returning home these people will take away with them for life the memory of the country and of the institutions in which they were trained. This will result in the arrival of other young people and in the selling of equipment and may have even greater political implications. Last but not least, many of the best will stay. In this context, there is no need to mention what the United States has gained by recruiting foreign scholars and scientists, often in their youth.

I hope this will not be considered as an undue intrusion into the affairs of a country where I of ten was and am currently a guest. Indeed, for the gratitude I have for what I learnt and received in your country I felt a moral obligation to express a disinterested opinion on what seems to me a very serious matter.

FIORENZOSTIRPE

Department of General Pathology,

University of Bologna,

I-40126 Bologna,

Italy 